\title{
Museo social en España: redes sociales y webs de los museos estatales
}

\author{
Social museum in Spain: Social networks and \\ websites of State museums
}

\author{
Florencia Claes; Luis Deltell
}

Cómo citar este artículo:

Claes, Florencia; Deltell, Luis (2019). "Museo social en España: redes sociales y webs de los museos estatales". El profesional de la información, v. 28, n. 3, e280304.

https://doi.org/10.3145/epi.2019.may.04

Artículo recibido el 21-09-2018

Aceptación definitiva: 16-02-2019

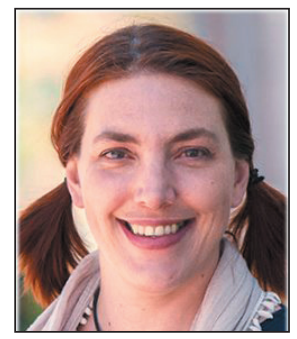

Florencia Claes $₫$

https://orcid.org/0000-0003-4291-0507

Universidad Rey Juan Carlos

Facultad de Ciencias de la Comunicación

Camino del Molino, s/n.

28943 Fuenlabrada (Madrid), España

florencia.claes@urjc.es

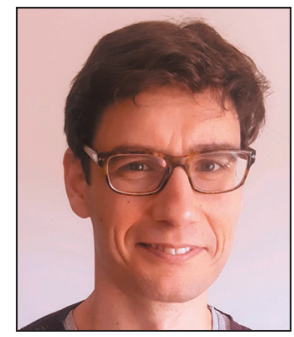

Luis Deltell

https://orcid.org/0000-0002-5230-1409

Universidad Complutense de Madrid Facultad de Ciencias de la Información Avenida Complutense, 3.

28040 Madrid, España

Ideltell@ccinf.ucm.es

\section{Resumen}

Se presentan las conclusiones de una investigación sobre la comunicación online de los diecinueve museos que pertenecen a la Dirección General de Bellas Artes de España (Museo Nacional del Prado, Museo Nacional Centro de Arte Reina Sofía y Museo Lázaro Galdiano más los dieciséis Museos Estatales dirigidos por la Subdirección General de Museos Estatales). Se monitorizó el uso de las redes sociales (Facebook, Instagram, Twitter y otras) y se analizaron las webs de estas instituciones. En la metodología se contabilizó el número de seguidores, el uso de idiomas, el volumen de publicaciones, el estudio de las webs, los backlinks de las mismas, así como el seguimiento de las actividades. Las conclusiones reflejan los buenos resultados en las redes sociales, especialmente Facebook, pero también la carencia en el uso de lenguas extranjeras y el acceso a datos de prensa y de transparencia en las webs. El Museo Nacional del Prado se muestra como el centro de referencia en comunicación online.

\section{Palabras clave}

Museos; Comunicación; Medios sociales; Redes sociales; Webs; Backlinks; Facebook; Twitter; Instagram; España.

\section{Abstract}

The conclusions of a research about the online communication of the nineteen museums belonging to the General Directorate of Fine Arts of Spain (Museo Nacional del Prado, Museo Nacional Centro de Arte Reina Sofía and Museo Lázaro Galdiano plus the sixteen Museos Estatales run by the Subdirección General de Museos Estatales) are presented. We monitored the use of social networks (Facebook, Instagram, Twitter and others) as well as the museum websites. We analized: number of followers, use of languages, volume of publications, web pages, backlinks, as well as the follow-up of

Financiación

Esta investigación ha sido posible gracias a la ayuda del Proyecto de Innovación Docente UCM núm. 278. Convocatoria $2018-2019$. Metodologías alternativas y colaborativas en el uso de la fotografía analógica en períodos de crisis II. Profesor Responsable: Luis Deltell.

Agradecimientos

Nuestro estudio habría sido imposible sin el apoyo de la Subdirección General de Museos Estatales, el Museo del Greco, Museo del Prado y muy especialmente el Museo Cerralbo. 
the online activities. The conclusions of our research show the good work done in significant aspects such as the content of the websites and some social network strategies (specially on Facebook), but also the lack of use of foreign languages, the difficult access to the press area, and transparency data in their websites. Museo Nacional del Prado is shown as the reference center in online communication.

\section{Keywords}

Museums; Communication; Social media; Social networks; Websites; Backlinks; Facebook; Twitter; Instagram; Spain.

\section{Introducción}

La museología crítica ha encontrado en internet, en las redes sociales y en los recursos digitales poderosas herramientas para construir un nuevo modelo de museo. Así lo reflejan algunos autores que han mostrado cómo las tecnologías de la información (TICS) posibilitaban un entorno social participativo. Fue el caso de Witcomb, que planteó esta revolución electrónica y el fin del museo-mausoleo (Witcomb, 1997) e, incluso, describió un centro de arte basado en trabajos comunicativos y no acumulativos (Witcomb, 2003). Esta nueva actitud de intercambio y de desarrollo de las TICs fue reforzada por teóricas como Simon (2010). Su experiencia en el Santa Cruz Museum of Arts le condujo a hablar de "un museo participativo, social y $2.0^{\prime \prime}$. Los museos no pueden ser mausoleos, sino que deben convertirse en espacios de co-creación entre las instituciones y los visitantes. Las TICs permiten romper con el dominio unidireccional de los organismos tradicionales, expandir estos centros más allá de sus entornos espaciales (Sookhanaphibarn; Chatuporn, 2013) y, además, establecer nuevos relatos hacia y con los visitantes físicos y virtuales (Kotler; Kotler; Kotler, 2008).

Desde principios del siglo XXI las investigaciones de Marty se centran en cómo las TICs cambian la percepción de la visita de los museos, primero en la transformación de la información y la distribución de contenidos (Marty, 2007a), pero también en cómo influye el discurso digital en el proceso previo y posterior a la visita física al centro (Marty, 2007b). Por tanto, la nueva estrategia de los museos se basa en crear espacios abiertos, donde el espectador pueda participar y construir. Así Sureda, del Museo Nacional Centro de Arte Reina Sofía (Mncars) sostenía:

"Si anteponemos el Museo como lugar de conocimiento y educación, donde se interpela al visitante y se le ofrecen herramientas para que construya sus propios relatos, estamos cumpliendo nuestra misión. Desviarse de nuestras líneas estratégicas o misión, cegados por la captación de más y más público, no consolidará el proyecto con sus propias especificidades" (Martínez-Peláez; Oliva-Marañón; Rodríguez-Rivas, 2012, p. 5).

Estos discursos se basan en una nueva comunicación y buscan la creación de un museo global y social, que pueda generar un espectador sin necesitar la proximidad física (Claes; Deltell, 2014), y permita la construcción de un discurso (o storytelling) digital y propio de cada visitante (Roussou et al., 2015).

Esta nueva comunicación y el nacimiento del museo social han sido unas de las claves de algunas investigaciones relevantes. Se ha analizado el uso de las webs de los centros de arte desde su aparición a finales del siglo XX hasta nuestros días (Sabin, 1997; Witcomb, 2003; Villaespesa, 2013). Destacan los trabajos sobre la visibilidad y las webs de los museos en Cataluña comenzados por Monistrol, Rovira y Codina (2006), continuados por Badell y Rovira (2010) y los últimos de Badell (2015). También se ha abordado el manejo de Twitter, desde los estudios de DosDoce (2009) que reflejaban que sólo el 15\% de los museos tenían cuenta en esta red en 2009, a los nuevos trabajos que muestran su empleo completamente generalizado (Caerols-Mateo; Viñarás-Abad; Gonzálvez-Valles, 2017).

Como bien han observado Capriotti y Losada-Díaz, la red social preferida por los internautas para la comunicación entre visitantes virtuales y museos es Facebook. Esta comunidad ha sido, al menos hasta principios de 2018, el lugar más activo. Los estudios de Losada-Díaz y Capriotti (2015) y Capriotti y Losada-Díaz (2018), revelan el buen comportamiento online de los museos españoles con más visitantes. Además, reflejan la gran actividad de estas instituciones en dicha red social.

Especial interés han cobrado las investigaciones que indagan en la construcción de un espacio virtual de encuentro, un museo plenamente social. Varios estudios ponen como referente de éste a la Tate Modern de Londres como espacio de intercambio virtual que entierra definitivamente la idea de colección estanca para convertirse en un lugar compartido y participativo online (Stack, 2010; 2013; Claes; Deltell; 2014). Incluso, como dice Gómez-Vílchez (2012), también se pude hablar de un museo social en algunos espacios situados en España.

Sin embargo, se observan algunos aspectos menos tratados en la construcción de este espacio virtual:

- el uso de las lenguas que, como indican Losada-Díaz y Capriotti (2015), se suele centrar sólo en los idiomas nativos de los países de los museos ( $y$, con cierta frecuencia, el apoyo de una versión reducida en inglés);

- no se ha analizado la red social Instagram, gracias a la cual el Prado ha logrado éxitos reseñables desde 2017;

- no se ha abordado el estudio de museos que compartan el carácter, la misión y los objetivos, como es el caso de los Museos Estatales. 


\section{Corpus, objetivos e hipótesis}

Nuestra investigación analiza la construcción de nuevos discursos y la participación digital en torno a los museos. Para ello aborda la comunicación online de los dieciséis centros pertenecientes a la Subdirección General de Museos Estatales. Además, se incluyen tres instituciones que quedan fuera de esta clasificación, por sus estatutos, pero que comparten estrategias y pertenecen a la Dirección General de Bellas Artes de España

Esta muestra tan amplia se debe a los siguientes motivos:

A. Pertenecen a la Dirección General de Bellas Artes de España, una misma institución, pública, abierta y no lucrativa, y desde la democracia estos espacios se entienden como "nuevos museos" (artículo 59.3, Ley 16/1985). Además, comparten ideario y misión comunes: la construcción de espacios participativos y entornos virtuales. Los dieciséis defienden el "decálogo de Museos+Sociales" (Subdirección General de Museos Estatales, 2017a) y forman parte del proyecto "Museos+Personas = Museos+Sociales" (Subdirección General de Museos Estatales, 2017b). Los tres restantes también comparten misión y se autodefinen como espacios de encuentro.

B. Los centros estudiados ofrecen el número de visitantes y objetivos. Esto permite no recurrir a datos menos precisos, como los publicados por Theartsnewspaper, Statista o prensa generalista, cuyas estadísticas contienen errores y cifras no verificadas.

C. Los centros de la selección tienen distintos tamaños, diferentes temáticas y gestionan presupuestos muy desiguales. Estos diecinueve fluctúan desde pequeños centros con 13.200 visitantes (Museo Casa de Cervantes en Valladolid) a los 3.880.812 del Mncars.

D. Se encuentran los dos museos con más visitas de España (Mncars y Prado) y los dos con más impacto social (los anteriores, pero en diferente orden).

E. España es el segundo país con mayor número de turistas extranjeros, más de 81 millones en 2017 (datos del Instituto Nacional de Estadística, INE, enero 2018) y con un importante porcentaje de turismo cultural. Esto nos permite analizar la construcción de un museo social fuera de la proximidad física.

\subsection{Objetivos}

La investigación se plantea estudiar la actividad de los museos en la Red. Para ello se analizan las estrategias de dichos espacios en las redes sociales, las webs y su impacto en número de seguidores.

1. Analizar el número total de visitantes físicos y virtuales en los años 2016, 2017 y primeros seis meses de 2018.

2. Monitorización de las webs de los diecinueve centros seleccionados. Estudio de contenidos y usabilidad de las mismas. Análisis de visibilidad contabilizando los backlinks o los enlaces de referencia que logran estos espacios virtuales y el número de visitas online.

3. Seguimiento y análisis del uso de los idiomas en redes sociales, webs y comunicación participativa.

4. Estudio y seguimiento de los perfiles oficiales de los centros en las redes sociales: Facebook, Instagram, Twitter, YouTube y otros, especialmente en la construcción de seguidores y visitantes virtuales.

En la tabla 1 se presentan los datos básicos de la selección de la muestra.

\subsection{Preguntas de investigación}

El estudio se plantea tres preguntas de investigación. Utilizamos un modelo derivado del trabajo de Losada-Díaz y Capriotti (2015).

PI1: Planteamiento. ¿Tienen los museos perfiles en las redes, cuál es el uso de los recursos de los museos online?

PI2: Presencia y actividad. ¿Existe una relación directa entre el número de visitantes físicos y el impacto social online de los museos?

PI3: Escucha y respuesta participativa. ¿Cuál es el impacto participativo en la red de los museos nacionales de España?

\subsection{Metodología}

a) En la monitorización de Facebook, Twitter e Instagram se han seguido los contadores públicos de estas redes y se ha utilizado el sistema T-hoarder para la comparación de flujos de tweets. Además, se han observado todos los perfiles oficiales para valorar la participación ofrecida y puntuación. T-hoarder es un sistema de monitorización de tweets por Mariluz Congosto, investigadora de la Universidad Carlos III de Madrid.

b) Se ha consultado el Boletín Oficial del Estado, los estatutos y la normativa de los diecinueve centros, para comprobar su misión y objetivos. Se ha revisado y medido la transparencia en datos de todos ellos.

c) Se ha hecho un análisis de las webs siguiendo el modelo propuesto por Sabin (1997), por Witcomb (2003) y Nielsen (2002). En esta investigación hemos puntuado de uno a cuatro los contenidos de los siguientes items: 
Tabla 1. Datos básicos del corpus

\begin{tabular}{|c|c|c|c|c|c|}
\hline \multirow[b]{2}{*}{ Nombre del centro } & \multirow[b]{2}{*}{ Ciudad } & \multirow[b]{2}{*}{ Tema central } & \multicolumn{3}{|c|}{ Visitantes } \\
\hline & & & 2016 & 2017 & $\begin{array}{c}\text { enero-julio } \\
2018\end{array}$ \\
\hline M. Arqueológico Nacional & Madrid & Arqueología y cultura española & 507.253 & 542.870 & 311.936 \\
\hline M. Casa de Cervantes & Valladolid & $\begin{array}{l}\text { En torno a la figura de Miguel de } \\
\text { Cervantes }\end{array}$ & 26.431 & 22.248 & 13.209 \\
\hline M. Cerralbo & Madrid & Colección Cerralbo & 130.364 & 136.098 & 59.841 \\
\hline M. de Altamira & Santillana del Mar & $\begin{array}{l}\text { Arte rupestre y gestión cuevas de } \\
\text { Altamira }\end{array}$ & 285.990 & 287.929 & 160.215 \\
\hline M. de América & Madrid & América precolombina y arte colonial & 80.345 & 84.060 & 62.974 \\
\hline M. del Greco & Toledo & El Greco & 236.887 & 261.220 & 139.994 \\
\hline M. del Traje & Madrid & Traje, moda y cultura del vestido & 108.177 & 102.605 & 85.456 \\
\hline M. Nacional de Antropología & Madrid & Antropología & 73.627 & 81.790 & 54.290 \\
\hline M. Nacional de Arqueología Subacuática & Cartagena & Arqueología submarina & 104.288 & 92.563 & 57.954 \\
\hline M. Nacional de Arte Romano & Mérida & Arte y cultura romanos & 226.591 & 239.798 & 134.680 \\
\hline M. Nacional de Artes Decorativas & Madrid & Arte decorativo & 34.892 & 71.472 & 79.244 \\
\hline $\begin{array}{l}\text { M. Nacional de Cerámica y Artes Suntua- } \\
\text { rias "González Marti" }\end{array}$ & Valencia & Cerámica & 166.286 & 183.320 & 108.097 \\
\hline $\begin{array}{l}\text { M. Nacional de Escultura (+ reproduccio- } \\
\text { nes artísticas) }\end{array}$ & Valladolid & Escultura y reproducción & 193.665 & 155.819 & 90.682 \\
\hline M. Nacional del Romanticismo & Madrid & Romanticismo & 97.791 & 97.667 & 54.504 \\
\hline M. Sefardí & Toledo & Legado hispanojudío y sefardí & 322.317 & 345.490 & 204.769 \\
\hline M. Sorolla & Madrid & Sorolla & 215.423 & 255.051 & 165.864 \\
\hline M. Nacional del Prado & Madrid & Arte hasta s.XIX & 3.033 .754 & 2.824 .404 & 1.728 .371 \\
\hline M. Nacional Centro de Arte Reina Sofía & Madrid & Arte contemporáneo & 3.646 .598 & 3.880 .812 & sin datos \\
\hline M. Lázaro Galdiano & Madrid & Colección Lázaro Galdiano & 60.573 & 64.101 & sin datos \\
\hline
\end{tabular}

Fuente: elaborado con datos de la Dirección General de Bellas Artes de España y las propias webs de los museos mencionados.

- visita (información práctica, mapas y horarios);

- colección/fondos y estudios e investigación (contenidos sobre análisis y trabajos del museo o sobre la colección);

- exposiciones temporales/actividades (información sobre las colecciones expuestas y recorridos posibles y datos generales, no se valoran las actividades en sí);

- prensa y comunicación y facilidad de acceso a medios sociales.

Por último, estudiamos la usabilidad.

También se ha registrado el contenido en idiomas.

d) Monitorización de visitas a las webs y seguimiento de backlinks (totales y referencia) contabilizados por SEMrush (sistema online que realiza auditorías SEO y SEM, análisis de la competencia y backlinks, entre otros).

\section{Resultados y discusión}

Antes de comenzar a abordar el estudio de los museos debemos presentar un dato relevante sobre las visitas físicas a éstos. Aunque hemos encontrado más de cien referencias en prensa generalista y más de veinte en revistas especializadas (entre ellas Theartsnewspaper) que señalan el Mncars como el museo con más visitas en España, este dato debe explicarse detenidamente. En realidad, los 3.880 .812 visitantes en 2017 no se deben a la sede central, sino a la suma de tres centros muy diferentes:

-el edificio central (1.659.986);

-el Palacio de Cristal (1.729.292);

-el Palacio de Velázquez (491.554),

estas dos últimas sedes situadas en el Parque del Retiro en Madrid. Como se observa, la sede central (edificios Sabattini y Nouvel) tiene un volumen muy inferior de visitantes que el Prado (2.824.404) y sólo se sitúa por encima al sumar las visitas al Palacio de Cristal y Palacio de Velázquez (2.320.856). Los espacios ubicados en el jardín madrileño, si bien pertenecen al Mncars, son periféricos y, sobre todo, gratuitos, lo que los convierte en casos muy excepcionales; incluso no se expenden entradas físicas, sino que el cómputo de visitantes se hace a mano. Aunque no es el tema central de esta investigación y, por ello, mantendremos el número de visitas oficiales, sí queremos abrir un debate científico sobre cómo se deben contabilizar las visitas físicas a los museos. 


\subsection{Web}

Sin duda, las webs de los museos son la puerta de entrada para la construcción del espacio virtual. Su desarrollo se ha considerado como pieza principal desde los primeros trabajos teóricos como los publicados por Sabin (1997) y Witcomb (1997). Todos los centros estudiados cuidan este medio y se han esforzado en su mantenimiento. Baste comparar la página inicial del Prado en 1997 y la misma veintiún años después (imagen 1 e imagen 2).

Los museos estatales utilizan el sistema de gestión Magnolia (Lorenzo-Jáudenes, 2015) aunque cada centro tiene potestad para incluir contenidos, información y para desarrollarla, dentro de las posibilidades del museo.

Ofrecemos un análisis de visibilidad basado en los backlinks, enlaces de entrada que logran las webs analizadas, contabilizados por SEMrush. Como sostienen Lopezosa y Codina los backlinks

"son los enlaces que recibe una página procedentes de otras páginas (...). La autoridad de un sitio se consigue por el número y la calidad de los enlaces de entrada o backlinks que consigue" (Lopezosa; Codina, 2017, pp. 16)
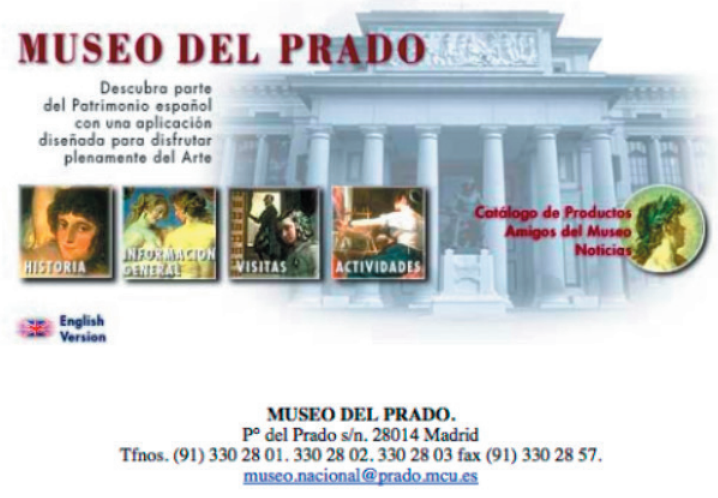

COPYRIGHT DEL MUSEO DEL PRADO

APLICACION DESARROLLADA POR ARROBA SYSTEMS

Última actualización: Diciembre 1997

Historia I Información General I Visitas I Actividades Catálogo de Productos I Amigos del Museo I Noticias

History I General Information I Visits I Activities Products Catalog I Eriends of the Museum I News

Imagen 1. Portada web Museo Nacional del Prado. Agosto 1997.

En nuestra monitorización tenemos en cuenta el número total y la referencia de calidad de éstos.

Tabla 2. Análisis de las webs de los museos

\begin{tabular}{|c|c|c|c|c|c|c|c|c|c|c|}
\hline \multirow[b]{2}{*}{ 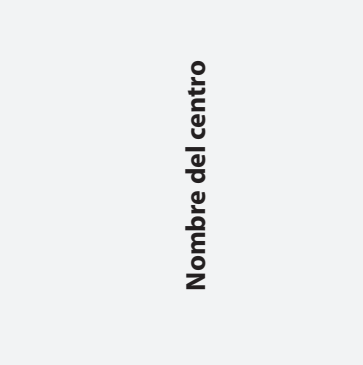 } & \multirow[b]{2}{*}{$\begin{array}{l}\stackrel{0}{\pi} \\
\text { हี } \\
\text { 윤 }\end{array}$} & \multirow[b]{2}{*}{$\frac{\frac{\pi}{n}}{5}$} & \multicolumn{3}{|c|}{ Contenidos } & \multirow[b]{2}{*}{ 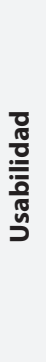 } & \multirow[b]{2}{*}{ 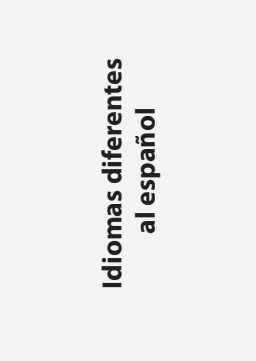 } & \multicolumn{3}{|c|}{ Análisis de visibilidad } \\
\hline & & & 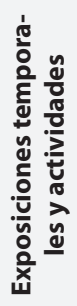 & 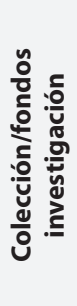 & 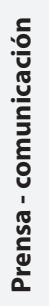 & & & 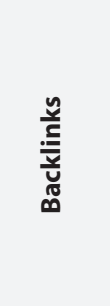 & 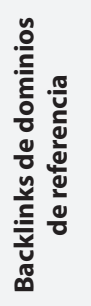 & 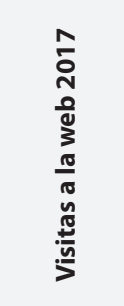 \\
\hline M. Casa de Cervantes & Plantilla & 2 & 2 & 2 & 1 & 2 & 0 & 30 & 5 & 11.015 \\
\hline M. N. Arqueología Subacuática & Plantilla & 3 & 3 & 3 & 1 & 2 & 0 & 738 & 28 & 24.470 \\
\hline M. de Altamira & Plantilla & 3 & 2 & 3 & 2 & 3 & PDFs en 3 idiomas & 316 & 60 & 283.370 \\
\hline M. N.A. Decorativas & Plantilla & 3 & 3 & 3 & 2 & 4 & Plano en 2 idiomas & 404 & 69 & 63.927 \\
\hline M. Sefardí & Plantilla & 3 & 3 & 3 & 2 & 3 & 0 & 1.797 & 217 & 24.522 \\
\hline M. del Greco & Plantilla & 3 & 2 & 3 & 2 & 2 & 0 & 2.291 & 243 & 35.450 \\
\hline M. N. Cerámica & Plantilla & 2 & 2 & 3 & 2 & 3 & PDF en valenciano & 3.703 & 321 & 31.968 \\
\hline M. N. Escultura & Plantilla & 3 & 3 & 3 & 1 & 3 & 0 & 3.678 & 327 & 37.263 \\
\hline M. N. Arte Romano & Plantilla & 3 & 2 & 3 & 1 & 2 & Plano en 2 idiomas & 22.070 & 363 & 35.989 \\
\hline M. N. de Antropología & Plantilla & 3 & 2 & 3 & 2 & 2 & 0 & 6.202 & 427 & 79.640 \\
\hline M. de América & Plantilla & 3 & 2 & 3 & 2 & 3 & 0 & 9.497 & 447 & 80.995 \\
\hline M. Cerralbo & Plantilla & 3 & 3 & 3 & 2 & 3 & PDFs en 3 idiomas & 8.396 & 470 & 84.878 \\
\hline M. N. del Romanticismo & Plantilla & 3 & 3 & 3 & 4 & 4 & Plano en 2 idiomas & 7.427 & 514 & 102.165 \\
\hline M. Sorolla & Plantilla & 4 & 3 & 3 & 2 & 4 & 0 & 14.298 & 840 & 215.752 \\
\hline M. del Traje & Plantilla & 4 & 4 & 3 & 4 & 3 & Plano en 2 idiomas & 28.738 & 947 & 120.459 \\
\hline M. Arqueológico Nacional & Plantilla & 4 & 4 & 3 & 3 & 4 & Inglés (parcial) & 30.704 & 2.021 & 432.709 \\
\hline M. Nacional del Prado & Personalizado & 4 & 4 & 4 & 4 & 4 & Inglés & 445.583 & 8.240 & 6.137 .079 \\
\hline M. N. Centro de Arte Reina Sofía & Personalizado & 4 & 4 & 4 & 4 & 4 & Inglés & 766.010 & 6.507 & 2.969 .058 \\
\hline M. Lázaro Galdiano & Personalizado & 4 & 3 & 3 & 4 & 3 & Portada en 7 idiomas & 38.959 & 872 & 131.904 \\
\hline
\end{tabular}


Un dato previsible es que las webs de los museos presentan un desnivel según los tamaños de los mismos. Frente a las webs del Prado y del Mncars, que logran la máxima puntuación en todos los aspectos, los espacios virtuales de los museos más pequeños presentan soluciones más modestas. Sin embargo, también se observan ciertas diferencias importantes entre centros de similar impacto. Así, el Prado mantiene una web más uniforme y con más contenido que la del Mncars, aunque su puntuación sea la misma. Entre los museos estatales se percibe cierta

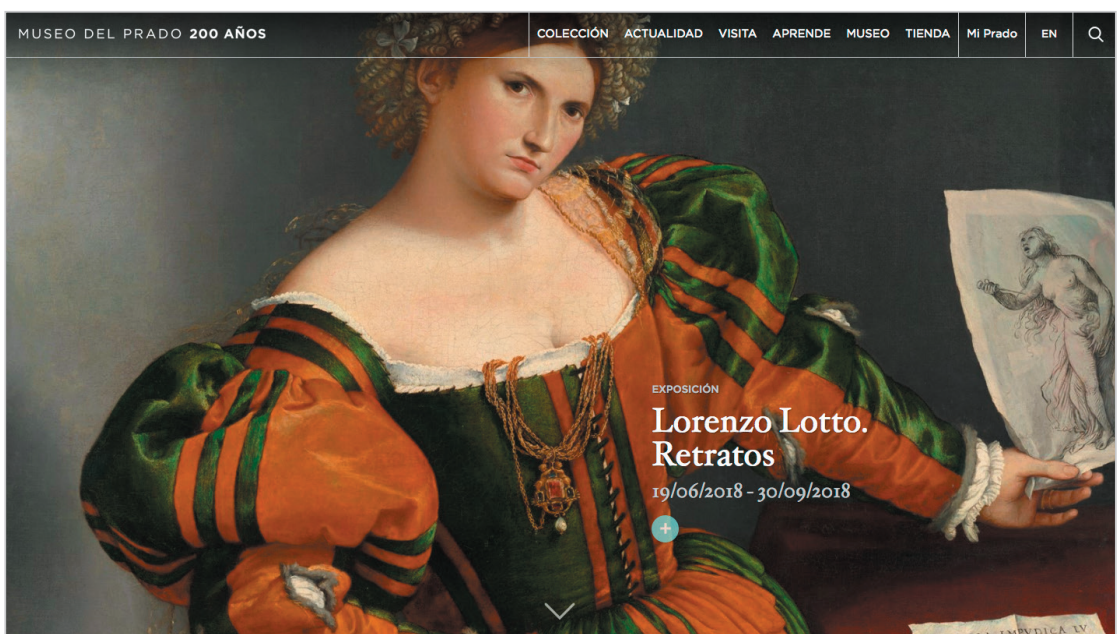

Imagen 2. Portada web Museo Nacional del Prado. Agosto 2018.

uniformidad, todos cumplen los aspectos mínimos y desarrollan una comunicación eficiente en la mayoría de los ítems estudiados. El uso del contenedor Magnolia se presenta como una solución práctica para lograr webs que cumplan con las necesidades básicas de las instituciones.

El número de visitantes a las webs revela el enorme impacto del Prado, que sobrepasa los seis millones de visitas. Supera en más del doble al siguiente museo. Este dato indica el gran interés de dicho espacio para los internautas. Entre los museos estatales, ninguno destaca especialmente en las visitas web, aunque sí existe una diferencia entre aquellos centros que tienen más visitantes virtuales que físicos (Museo Arqueológico Nacional, Museo del Traje y Museo del Romanticismo) y, por otro lado, los que tienen menos visitas web que presenciales (M. N. de Escultura y M. N. de Cerámica).

Las carencias más significativas se encuentran en los aspectos de comunicación/prensa y en el uso de idiomas extranjeros. Sorprende que muchos centros no tengan contenidos (o descripciones mínimas) en inglés u otros idiomas (muy especialmente el francés o el portugués en los museos limítrofes con estos países, como el Museo Nacional de Arte Romano en Mérida).

Al observar los backlinks se detecta de nuevo el predominio de los grandes. Un dato muy relevante es que el Prado obtiene significativamente más enlaces de entradas de referencia y calidad. También se resalta que los museos estatales que no ofrecen una buena sección de prensa y comunicación tienden a tener menos backlinks de referencia.

Se presenta un aspecto negativo en la baja transparencia de información de datos relevantes (presupuestos, visitantes...). Es cierto que los centros dependientes de Subdirección General de Museos Estatales ofrecen estadísticas comunes en la página central de dicha Subdirección, pero el acceso no es sencillo y no se relaciona directamente con las sedes. Todos ellos firman el proyecto digital y social del Decálogo de Museos+Sociales (Subdirección General de Museos Estatales, 2017a) y su extensión en “Museos+Personas = Museos+Sociales (Subdirección General de Museos Estatales, 2017b). En estos documentos se perfilan los planes digitales estratégicos para la construcción de un museo social.

\subsection{Redes sociales}

Como se indicó en los antecedentes, Facebook es la red preferida para la comunicación entre museos y ciudadanos. En nuestro estudio se percibe una gran actividad por parte de los centros, sin embargo, el número de seguidores en este medio (1.690.000) es menor que en Twitter (donde llegan a más de 2.360.000). Los museos que tienen más actividad vuelven a ser el Prado, que ya había sido estudiado por Viñarás-Abad y Cabezuelo-Lorenzo (2012), y el Mncars. Dentro de los estatales se encuentran diferencias significativas. El Museo del Traje y el Museo Sorolla tienen un volumen de seguidores superior a lo que les correspondería por su cantidad de visitantes físicos.

Un dato interesante es la puntuación que los seguidores de Facebook dan a los museos españoles. Todos ellos obtienen calificaciones altas, en las que destaca con 4,8 el Museo Arqueológico Nacional, que parece beneficiarse de su reciente reforma integral.

En el caso de Twitter de nuevo destaca el Prado por número de seguidores y por la rapidez en responder a éstos (en nuestro experimento tardaron menos de doce horas). Además de este centro el Museo Lázaro Galdiano logra más de 80.000 seguidores. También resulta destacable la generación de comunidad que intentan los perfiles del Cerralbo, del Romanticismo y del Traje. El centro de Arqueología Subacuática, creó su perfil en mayo de 2018. Sólo un museo no tiene cuenta oficial en Twitter y es el de Antropología.
Facebook e Instagram son las dos redes sociales con más crecimiento en el entorno museístico 
Una de las redes sociales menos estudiadas en el caso museístico es Instagram. Su impacto en número de seguidores es mucho menor que los anteriores. Sin embargo, desde 2017 el Sorolla y el Prado han realizado un trabajo de generación de comunidad excelente. El primero de ellos parece beneficiarse de la exposición sobre Sorolla y la moda celebrada entre febrero y mayo de 2018. El Prado en agosto de 2017 comenzó a realizar transmisiones de vídeos en directo a través de Instagram, es decir, pequeñas explicaciones audiovisuales de las piezas de la colección, que se visualizan desde el móvil o la tablet. En el período estudiado, el crecimiento de su perfil oficial es superior a 1.500 seguidores diarios, y actualmente, supera ya los 300.000 (septiembre 2018).

Tabla 3. Análisis de las redes sociales

\begin{tabular}{|c|c|c|c|c|c|c|c|c|c|c|c|c|}
\hline \multirow[b]{2}{*}{ Nombre del centro } & \multicolumn{3}{|c|}{ Twitter } & \multicolumn{3}{|c|}{ Facebook } & \multicolumn{2}{|c|}{ Instagram } & \multicolumn{4}{|c|}{ YouTube } \\
\hline & 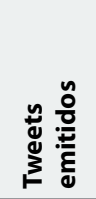 & 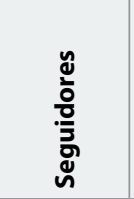 & $\begin{array}{l}\frac{0}{2} \\
\frac{1}{2} \\
\frac{0}{2}\end{array}$ & 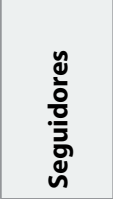 & 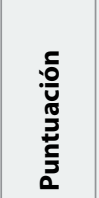 & $\begin{array}{l}\text { y } \\
\frac{0}{c} \\
\frac{\bar{c}}{0}\end{array}$ & 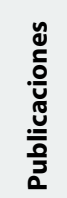 & $\begin{array}{l}\text { y } \\
\frac{0}{0} \\
\frac{0}{3} \\
\text { ஸे }\end{array}$ & 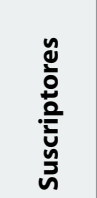 & 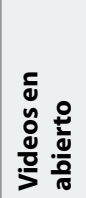 & 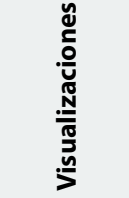 & $\begin{array}{l}\frac{0}{3} \\
\frac{2}{2} \\
\frac{0}{2}\end{array}$ \\
\hline M. Arqueológico Nacional & 13.478 & 42.777 & $4 / 4 / 14$ & 53.297 & 4,8 & 794 & no & no & 3.190 & 847 & 338.815 & $20 / 4 / 14$ \\
\hline M. Casa de Cervantes & 1.445 & 10.624 & $16 / 3 / 12$ & 2.636 & sin inf. & sin inf. & 8 & 85 & no & no & no & no \\
\hline M. Cerralbo & 23.569 & 30.448 & $29 / 10 / 10$ & 11.964 & 4,6 & 505 & 346 & 9.174 & sin inf. & 31 & 4.868 & $10 / 7 / 15$ \\
\hline M. de Altamira & 7.056 & 10.468 & $20 / 2 / 14$ & 19.165 & 4,5 & 484 & 175 & 2.058 & 862 & 72 & 364.309 & $2 / 2 / 12$ \\
\hline M. de América & 3.207 & 7.954 & $7 / 4 / 12$ & 11.280 & 4,3 & 745 & no & no & 3.982 & 362 & 1.794 .282 & $3 / 11 / 11$ \\
\hline M. del Greco & 4.621 & 15.769 & $26 / 4 / 11$ & 6.333 & 4,4 & 159 & 65 & 1.414 & no & no & no & no \\
\hline M. del traje & 6.699 & 23.793 & $2 / 8 / 11$ & 64.376 & 4,5 & 1.279 & 181 & 11.843 & 13 & 22 & 5.111 & 2011 \\
\hline M. N. de Antropología & 449 & 71 & $17 / 10 / 16$ & 18.102 & 4,4 & 498 & 64 & 1.448 & 30 & 5 & 2.974 & $20 / 12 / 17$ \\
\hline M. N. Arqueología Subacuática & 83 & 176 & $17 / 5 / 18$ & 11.071 & 4,6 & 69 & 84 & 1.096 & no & no & no & no \\
\hline M. N. Arte Romano & 1.852 & 16.640 & $14 / 3 / 11$ & 23.577 & 4,6 & 1.016 & no & no & 144 & 10 & 1.163 & $18 / 1 / 18$ \\
\hline M. N. A. Decorativas & 4.726 & 17.635 & $6 / 10 / 11$ & 14.927 & 4,4 & 375 & 501 & 2.656 & $\mathrm{~s} / \mathrm{i}$ & 86 & 12.453 & $23 / 5 / 13$ \\
\hline M. N. Cerámica & 2.168 & 1.760 & $20 / 4 / 12$ & 5.707 & 4,5 & 289 & no & no & no & no & no & no \\
\hline M. N. Escultura & 7.033 & 15.093 & $7 / 3 / 12$ & 8.935 & 4,7 & 221 & no & no & no & no & no & no \\
\hline M. N. del Romanticismo & 27.039 & 49.471 & 19/9/11 & 16.153 & 4,5 & 705 & 930 & 12.558 & 238 & 49 & 22.396 & $13 / 12 / 11$ \\
\hline M. Sefardí & 3.377 & 11.194 & $2 / 2 / 11$ & 10.173 & 4,7 & 87 & 99 & 1.000 & 51 & 12 & 124 & sin inf. \\
\hline M. Sorolla & 3.215 & 49.082 & $24 / 3 / 11$ & 51.885 & 4,7 & 1.832 & 718 & 50.768 & no & no & no & no \\
\hline M. Nacional del Prado & 46.707 & 1.226 .920 & $3 / 10 / 08$ & 957.235 & 4,7 & 47.515 & 305 & 300.982 & 34.385 & 1.485 & 4.458 .277 & $6 / 3 / 07$ \\
\hline M. N. C. A. Reina Sofía & 21.219 & 752.046 & $1 / 2 / 09$ & 367.363 & 4,5 & 19.261 & 652 & 58.258 & 4.320 & 161 & 406.639 & $5 / 4 / 09$ \\
\hline M. Lázaro Galdiano & 20.677 & 80.218 & $12 / 5 / 11$ & 6.739 & 4,6 & 47 & 458 & 5.429 & 255 & 40 & 48.733 & $31 / 1 / 12$ \\
\hline
\end{tabular}

Pinterest, Spotify y Flickr tienen unos resultados residuales comparados con las tres comunidades mencionadas previamente. También las cuentas oficiales museísticas en YouTube alcanzan muy pocos seguidores. Sin embargo, en este caso, dos museos logran un importante número de visualizaciones: el Prado (4.458.277) y el Museo de América, que alcanza la cifra de 1.794.282. Como hemos visto, la pinacoteca es siempre el museo social con más impacto, no obstante, el Museo de América tiene perfiles bajos, pero logra una cifra elevada en YouTube. Esto se debe a que sus vídeos versan sobre tradiciones, experiencias y bailes de América Latina, permitiendo la creación de una comunidad online que comparte dichos intereses. También resultan destacables acciones del tipo \#museundanceoff, en la que participó el Museo de Escultura, con buena acogida en YouTube.

Un dato a destacar, que se repite en otras monitorizaciones, es el cuidado del Museo del Romanticismo que si bien no logra las primeras posiciones en número de seguidores, sí está presente activamente en todas las redes sociales y muestra una coherencia de estilo en ellas. Este espacio ya ha sido comentado por otras investigaciones (Cabrera-Bravo; Cabrejas-Almena, 2013), (Viñarás-Abad, 2014) y (Claes; Deltell, 2014) como un ejemplo de museo pequeño (97.667 visitantes físicos en 2017) pero con una esmerada y excelente comunicación en redes sociales. También en nuestro experimento destacamos la buena estrategia del Cerralbo, que en los últimos años ha sabido plantear una nueva comunicación online atendiendo de forma esmerada sus perfiles en los medios sociales.

\subsection{Otras estrategias}

Una de las posibilidades de las TICs es el uso de los móviles para elaborar discursos museísticos y mejorar la experiencia de la visita física y virtual. Se ha constatado en diversas investigaciones el beneficio de la utilización de apps, ya sea como guías digitales de los museos, realidades aumentadas (Romano et al., 2016) o procesos de ludificación o gamificación de los mismos (Coenen; Mostmans; Naessens, 2013; Paliokas; Sylaiou, 2016). Estas herramientas, según estudios recientes, se dirigen tanto al apoyo de visitas familiares como de adultos y expertos (Rennick-Egglestone et al., 2016). 
De los museos estatales, únicamente cinco ofrecen apps o guías virtuales de descarga en la web. Éstas son explicaciones y recorridos dirigidos sobre las colecciones. Están diseñadas básicamente para la visita física y son desiguales en su desarrollo. El de América muestra un recorrido con realidad aumentada y el Museo Arqueológico ofrece dos guías oficiales, una de ellas para personas con discapacidad visual. Además, este museo realiza actividades de realidad virtual. También el Lázaro Galdiano ofrece un juego interactivo llamado Enigma.

Sin embargo, ninguno de ellos ofrece apps participativas o de elaboración de discursos propios sobre la colección expuesta. Los dos museos más grandes tienen diversas apps y guías virtuales oficiales. Como se ha visto en las redes sociales, el Prado ha logrado no sólo crear una comunicación horizontal virtual sólida, sino que la participación ciudadana es muy alta. También en las otras estrategias, este centro se muestra más innovador y participativo. Su proyecto Mi Prado es un buen intento de crear un espacio de intercambio. De una manera lúdica permite la reconstrucción de un discurso museístico propio sobre la pinacoteca. Además, este relato/recorrido se puede compartir con la comunidad de visitantes virtuales. Es, sin duda, el proyecto más completo y ambicioso de los monitorizados.

\section{Conclusiones}

El primer dato importante es el gran esfuerzo de los museos estatales por construir espacios sociales y compartidos en entornos virtuales a pesar de su escaso presupuesto. Todos los museos analizados tienen webs actualizadas, utilizan los medios sociales más populares y presentan actitudes y comportamientos digitales positivos. Además, los propios centros muestran de forma pública su decálogo de transparencia y buenas conductas y su misión social. Por lo tanto, la pregunta de investigación PI1 se responde positivamente, los museos sí tienen perfiles en las redes sociales y usan gran cantidad de recursos online.

La pregunta PI2 de nuestra investigación versa sobre si existe una relación entre visitantes físicos y visitantes virtuales. Esta parece ser cierta. Los museos con más entradas computadas son, también, los que tienen más seguidores (es importante recordar que la cifra del Mncars no se refiere a su sede central). Sin embargo, algunos museos logran tener más seguidores virtuales de lo que les correspondería por su número de visitantes físicos.

Para contestar a la pregunta PI3, escucha y respuesta participativa, la hemos abordado desde diversas perspectivas. Se puede entender que sí se produce una escucha, pero la respuesta participativa es desigual, según el museo y el medio utilizado. Así, el formato de web más usado por los museos estatales es el contenedor Magnolia. Este presenta algunas virtudes, para gestión y uniformidad de contenidos pero, a la vez, ofrece limitaciones y problemas para la presentación de las obras, las colecciones propuestas Todos los museos estudiados ofrecen webs con la información básica necesaria para la visita o las actividades del centro o de los visitantes. Las webs del Museo Arqueológico y el Mncars destacan por su cuidado y por su puntuación. Sin embargo, es el Prado el centro que presenta la web más completa tanto en calidad técnica como en contenidos. El estudio de los backlinks y de las visitas virtuales nos permite aventurar que la calidad de estos contenidos y la usabilidad de las webs ejercen una gran influencia en su visibilidad.

De las redes sociales destaca Facebook como la más empleada y que ha conseguido grandes logros (especialmente en números de seguidores) en la mayoría de los centros. El Prado y el Museo del Romanticismo ofrecen una relectura virtual de sus instalaciones; sus perfiles en este medio social son especialmente activos dentro de la comunidad online.

El aspecto más negativo que se encuentra en el estudio es la falta de uso de idiomas en los museos. En un país con más de 81 millones de turistas extranjeros al año y que durante dos temporadas seguidas es el segundo más visitado del mundo, no resulta comprensible esta situación. Salvo los grandes centros, los demás espacios no ofrecen información completa en otra lengua.

El museo español con mayor impacto virtual es el Prado. Es el más seguido en todos los medios sociales estudiados y su web se puede entender como un referente indiscutible del buen hacer virtual, de la transparencia y de la construcción de un espacio compartido y colaborativo. Iniciativas como las propuestas de directos en Instagram desde 2017 o Mi Prado lo convierten en un museo abierto y virtual. Además, sus visitantes se muestran participativos y activos, con medios sociales y apps, creando sus propios discursos en torno a este museo.

\section{Referencias}

Badell, Joan-Isidre (2015). "Los museos de Cataluña en las redes sociales: resultados de un estudio de investigación". Revista interamericana de bibliotecología, v. 38, n. 2, pp. 159-164. https://doi.org/10.17533/udea.rib.v38n2a07

Badell, Joan-Isidre; Rovira, Cristòfol (2010). "Visibilidad de las sedes web de los museos de Cataluña". Revista española de documentación científica, v. 33, n. 4, pp. 531-552.

https://doi.org/10.3989/redc.2010.4.754 
Cabrera-Bravo, María-Jesús; Cabrejas-Almena, María-Carmen (2013). “Del XIX al XXI: el Museo del Romanticismo en internet". Revista de museología, n. 56, pp. 88-100.

Caerols-Mateo, Raquel; Viñarás-Abad, Mónica; Gonzálvez-Valles, Juan-Enrique (2017). “Redes sociales y museos: análisis de la campaña en Twitter para el Día Internacional de los Museos y Noche de los Museos". Revista latina de comunicación social, v. 72, pp. 220-234.

https://doi.org/10.4185/RLCS-2017-1162

Capriotti, Paul; Losada-Díaz, José-Carlos (2018). "Facebook as a dialogic communication tool at the most visited museums of the world". El profesional de la información, v. 27, n. 3, pp. 642-650.

https://doi.org/10.3145/epi.2018.may.17

Claes, Florencia; Deltell, Luis (2014). “Museos sociales. Perfiles museísticos en Twitter y Facebook 2012-2013”. El profesional de la información, v. 23, n. 6, pp. 594-602.

https://doi.org/10.3145/epi.2014.nov.06

Coenen, Tanguy; Mostmans, Lien; Naessens, Kris (2013). “MuseUs: Case study of a pervasive cultural heritage serious game". Journal of computing and cultural heritage, v. 6, n. 2, article n. 8.

https://doi.org/10.1145/2460376.2460379

DosDoce; Abanlex Abogados (2009). La visibilidad de los museos en la Web 2.0.

http://www.dosdoce.com/upload/ficheros/noticias/201005/0000000048.pdf

Gómez-Vílchez, Soledad (2012). "Museos españoles y redes sociales". Telos, cuadernos de comunicación e innovación, n. 90.

https://telos.fundaciontelefonica.com/archivo/numero090/museos-espanoles-y-redes-sociales

Kotler, Neil G.; Kotler, Philip; Kotler, Wendy I. (2008). Museum marketing and strategy: designing missions, building audiences, generating revenue and resources. San Francisco: John Wiley \& Sons. ISBN: 9780787996918

Lopezosa, Carlos; Codina, Lluís (2017). “Análisis de posicionamiento en medios de comunicación con herramientas SEO. Cobertura informativa de los premios Oscar 2017". Informes DigiDoc-EPI, n. 2. Barcelona: Universitat Pompeu Fabra, Departamento de Comunicación; Ediciones Profesionales de la Información SL. ISBN: 9788469787984 http://www.elprofesionaldelainformacion.com/documentos/Analisis_posicionamiento_medios_comunicacion.pdf

Lorenzo-Jáudenes, Marta (2015). “Museos y redes sociales: el caso Museo Cerralbo”. Myartdiary.com, 14 abril. http://myartdiary.com/museos-redes-sociales-museo-cerralbo

Losada-Díaz, Juan-Carlos; Capriotti, Paul (2015). “La comunicación de los museos de arte en Facebook. Comparación entre las principales instituciones internacionales y españolas". Palabra clave, v. 18, n. 39, pp. 889-904.

https://doi.org/10.5294/pacla.2015.18.3.11

Martínez-Peláez, Agustín; Oliva-Marañón, Carlos; Rodríguez-Rivas, Ana-María (2012). “Interacción del público en un entorno virtual, Comunicación interna y externa en el Museo Reina Sofía”. Telos: Cuadernos de comunicación e innovación, n. 90, pp. 71-78.

https://telos.fundaciontelefonica.com/archivo/numero090/comunicacion-interna-y-externa-en-el-museo-reina-sofia

Marty, Paul F. (2007a). "The changing nature of information work in museums". Journal of the American Society for Information Science and Technology, v. 58, n. 1, pp. 97-107.

https://doi.org/10.1002/asi.20443

Marty, Paul F. (2007b). "Museum websites and museum visitors: Before and after the museum visit". Museum management and curatorship, v. 22, n. 4, pp. 337-360.

https://doi.org/10.1080/09647770701757708

Monistrol, Ricard; Rovira, Cristòfol; Codina, Lluís (2006). "Sitios web de museos de Cataluña: Análisis y propuesta de evaluación". Hipertext.net, n. 4.

https://www.upf.edu/hipertextnet/numero-4/museos.html

Nielsen, Jacob (2002). Usabilidad: diseño de sitios web. Madrid: Prentice-Hall. ISBN: 9788420530086

Paliokas, Ioannis; Sylaiou, Stella (2016). "The use of serious games in museum visits and exhibitions: A systematic mapping study". In: $8^{\text {th }}$ Intl conf on games and virtual worlds for serious applications, VS-Games 2016.

https://doi.org/10.1109/VS-GAMES.2016.7590371

Rennick-Egglestone, Stefan; Brundell, Patrick; Koleva, Boriana; Bendord, Steve; Roussou, Maria; Chaffardon, Christophe (2016). "Families and mobile devices in museums: designing for integrated experiences". Journal on computing and cultural heritage, v. 9, n. 2, pp. 1-13.

http://eprints.nottingham.ac.uk/32072/

https://doi.org/10.1145/2891416 
Romano, Marco; Díaz, Paloma; Aedo, Ignacio; D’Agostino, Pierpaolo (2016). “Augmenting smart objects for cultural heritage: A usability experiment". In: $3^{\text {rd }}$ Intl conf. augmented reality, virtual reality, and computer graphics, AVR 2016, Lecce, Italy, June 15-18. Part II, pp. 186-204. ISBN: 9783319406510

https://doi.org/10.1007/978-3-319-40651-0_15

Roussou, Maria; Pujol, Laia; Katifori, Akrivi; Chrysanthi, Angeliki; Perry, Sara; Vayanou, Maria (2015). "The museum as digital storyteller: Collaborative participatory creation of interactive digital experiences". In: MW2015: Museums and the Web 2015.

https://mw2015.museumsandtheweb.com/paper/the-museum-as-digital-storyteller-collaborative-participatorycreation-of-interactive-digital-experiences

https://doi.org/10.13140/RG.2.1.3917.2889

Sabin, Richard (1997). "Museums and their websites: An examination and assessment of how museums are coping with the challenge of the world wide web". Journal of conservation and museum studies, v. II, pp. 6-10.

https://doi.org/10.5334/jcms.2972

Simon, Nina (2010). The participatory museum. Santa Cruz, California: Museum 2.0.

http://www.participatorymuseum.org/read

Sookhanaphibarn, Kingkarn; Chatuporn, Utaiwan (2013). "Expanding the experience of museum visitors with a social application on Facebook". In: ICDS 2013: 7th Int/ conf on digital society, pp.74-80. ISBN: 9781612082493

https://www.thinkmind.org/download.php?articleid=icds_2013_3_40_10179

Stack, John (2010). "Tate online strategy 2010-2012". Tate papers.

http://www.tate.org.uk/research/publications/tate-papers/tate-online-strategy-2010-12

Stack, John (2013). "Tate digital strategy 2013-15: Digital as a dimension of everything". Tate papers.

http://www.tate.org.uk/research/publications/tate-papers/tate-digital-strategy-2013-15-digital-dimension-everything

Subdirección General de Museos Estatales (2017a). Museos + Sociales. Génesis de un plan destinado a reforzar el compromiso social de los museos, p. 251.

https://www.mecd.gob.es/dam/jcr:b615b103-3fad-428b-97d2-cdf8352a718c/01-museos-sociales.pdf

Subdirección General de Museos Estatales (2017b). Museos + Sociales. Un mayor compromiso social de los museos de la Secretaría de Estado de Cultura.

http://www.culturaydeporte.gob.es/dam/jcr:1550881a-1d02-4fe3-b06a-53cfeeb92de0/dossier.pdf

Villaespesa, Elena (2013). "El museo en la web social y su impacto en los visitantes". Revista de museología, v. 56, pp. $5-61$.

Viñarás-Abad, Mónica (2014). “Identidad corporativa y museos: los casos del Museo de Artes Decorativas, Museo Cerralbo, Museo Lázaro Galdiano, Museo del Romanticismo y Museo Sorolla”. En: Rodríguez-Terceño, José; Fernández-Paradas, Antonio (coords.). Nuevas culturas y sus nuevas lecturas, pp. 707-720. ISBN: 9788448197407

Viñarás-Abad, Mónica; Cabezuelo-Lorenzo, Francisco (2012). “Claves para la participación y generación de contenido en las redes sociales: estudio de caso del Museo del Prado en Facebook". adComunica, n. 3, pp. 87-103.

https://doi.org/10.6035/2174-0992.2012.3.6

Witcomb, Andrea (1997). "The end of the mausoleum: Museums in the age of electronic communication". In: Museums and the Web: An intl conf, Los Angeles, CA, March 16-19.

https://www.museumsandtheweb.com/mw97/speak/witcomb.html

Witcomb, Andrea (2003). Re-imagining the museum: beyond the mausoleum. Routledge: Londres. ISBN: 9780415220996

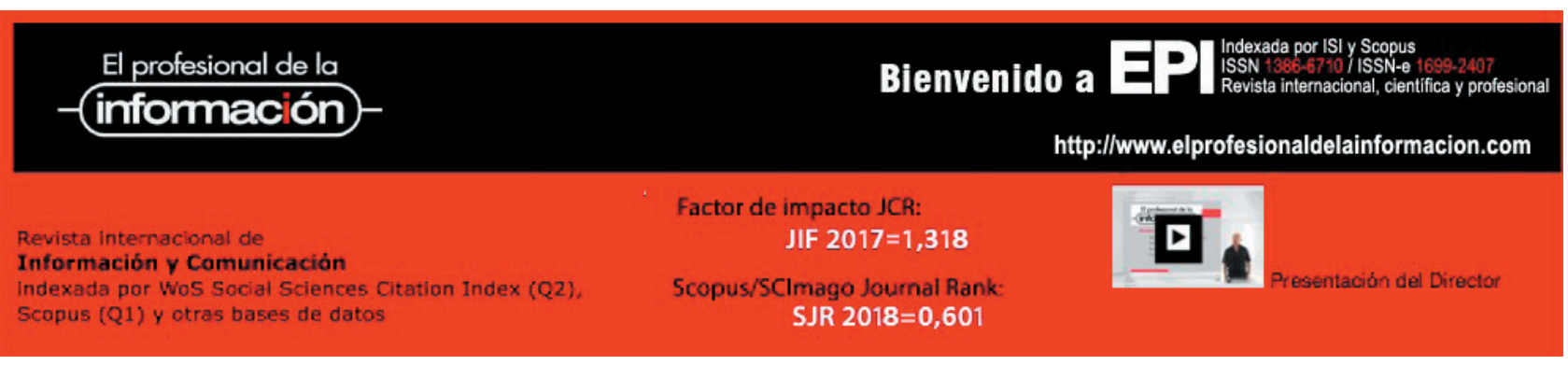

